Formseite im Verhältnis zur Aussagenseite nicht vernachlässigen. Da Einschlägiges im einleitenden Abschnitt durchaus reflektiert, die Aufmerksamkeit zugleich aber dezidiert auf »Handlungslogik«, »Figurenzeichnung « und die »Normwelten des Textes « (S. 6) gerichtet wird, kann man der insgesamt sehr ertragreichen und anregend argumentierenden Untersuchung darin freilich kaum ein Manko ankreiden. Ihr >Ausblickı eröffnet vielmehr im besten Sinne Möglichkeiten des Anschließens und Weiterdenkens.

\title{
Bernd Bastert, Andreas Bihrer u. Timo Reuvekamp-Felber (Hgg.): Mäzenaten im Mittelalter aus europäischer Perspektive. Von historischen Akteuren zu litera- rischen Textkonzepten, Göttingen: V\&R unipress 2017, 302 S., 7 Abb. (Encomia Deutsch 4)
}

Besprochen von Prof. Dr. Martin Schubert: Universität Duisburg-Essen, Institut für Germanistik, Berliner Platz 6, D-45127 Essen, E-Mail: Martin.Schubert@uni-due.de

https://doi.org/10.1515/bgsl-2018-0046

Ausgangspunkt des Sammelbands ist eine treffende Feststellung: Aus der für vormoderne Literatur wesentlichen Trias `Autor - Werk - Mäzen` wurden in neuerer Zeit zwei Begriffe ausführlich problematisiert, während der Bereich >Gönner/ Mäzen/Auftraggeber bislang unterrepräsentiert ist. Der auf eine Kieler Tagung von 2016 zurückgehende Band soll hier abhelfen.

Timo Reuveka m p-Felber (S. 9-22) erläutert in der >Einleitung`, wie das Thema seit Joachim Bumkes grundlegender Studie `Mäzene im Mittelalter ${ }^{1}$ vor allem sozialgeschichtlich wahrgenommen wurde, wobei die seit dem 13. Jahrhundert herausgehobene Funktion der weltlichen Fürsten im Zentrum des Interesses stand. Nun wird dazu aufgefordert, Gönnernennungen nicht vorrangig als Indikatoren historischer Zusammenhänge, sondern verstärkt in ihrer Funktion als Literarisierungsstrategien wahrzunehmen. Benannt werden als wichtige, interdisziplinär anzugehende Fragen der Umgang mit Gönnerverhältnissen im Vergleich zu Nachbarsprachen und zum Lateinischen, der Bezug zu kulturellen Hintergründen sowie die bildmediale Darstellung (vgl. S. 14). Der Band ist entsprechend in sich weiternden Kreisen gegliedert: in Grundlagenstudien, germanistische Fallstudien, Beiträge anderer Philologien und anderer Fächer. In der ersten und vierten Gruppe findet sich also, was bei der Ordnung nach Philologien übrigblieb.

1 Joachim Bumke: Mäzene im Mittelalter. Die Gönner und Auftraggeber der höfischen Literatur 1150-1300, München 1979. 
Andreas Bihrer (S. 25-51) fragt nach >Perspektiven für die Geschichtswissenschaft ‘ und vergleicht zunächst die geschichtswissenschaftliche und die altgermanistische Forschungsgeschichte. Historiker reagierten bislang reserviert auf den sozialgeschichtlichen Fokus und die Konzentration auf die Künstlerrolle, wie sie in germanistischen Arbeiten vorliegen. Bihrer erörtert ausführlich die Möglichkeiten wechselseitiger Anregungen in den Bereichen von Terminologie, Quellenlage, Akteuren, Interessen, Strukturen und historischem Wandel, wobei er zahlreiche noch offene Fragen benennt. Insgesamt handelt es sich um einen intensiven Appell zur Forschungsarbeit und Forschungskooperation, um letztlich genauer bestimmen zu können, welche Bedeutung Mäzenatentum im Mittelalter hatte und was daran typisch mittelalterlich war.

Jürgen Wolf (S. 53-66) lenkt den Blick auf die in der Forschung »zu Unrecht >vernachlässigten«« (S. 55) geistlichen Auftraggeber. Geistliche Eliten werden als Trägerschaft des Neubeginns deutscher Literatur im 11. Jahrhundert herausgestellt; als Publikum werden hier »rudimentär geistlich Gebildete, heilsbegierige Laien und nicht zuletzt [...] Frauen« (S. 57) angenommen. Dass die Ministerialität als »kulturelle Schnittstelle par excellence« (S. 58) beteiligt war, lässt sich für die Frühzeit nur vermuten. Wolf listet geistliche Auftraggeber des 13. Jahrhunderts auf, die durchaus auch weltliche Literatur förderten, wenn auch keine höfische. Da sich die konkrete Buchgestalt durchgehend aus dem geistlichen Schriftdiskurs speise, seien die geistlichen Förderer sehr wichtig, auch wenn ihre Rolle im Einzelnen schlecht nachzuzeichnen sei. Es folgt noch ein Ausblick auf geistliche Mäzene im Druckzeitalter, anhand einer in der Tat »höchst unvollständig[en] und höchst zufällig[en] [...] Beispielreihe« (S. 66), die nur zwei Beispiele umfasst.

Klaus Kipf (S. 67-102) behandelt Auftraggebernennungen in der zweiten Hälfte des 12. Jahrhunderts. Ausgehend von den Selbstnennungen Hartmanns von Aue zeigt er Traditionslinien auf, die in die frühmittelhochdeutsche geistliche Literatur zurückweisen. Vergleichend wird dann nach den Diskurstraditionen gefragt, in denen Selbst- und Gönnernennungen auftauchen. Eingehend bearbeitet wird dies an einer Reihe geistlicher Werke der Schwellenzeit (Albers `Tnugdalus`, Priester Wernhers `Maria`, >Rolandslied`, Veldekes `Sente Servas` u. a.). Gönnernennungen sind deutlich seltener als Autornennungen, und sie sind weniger standardisiert als diese. Feststellbar sind aber Diskurstraditionen wie die Bitte um Gebetsgedenken für beide Parteien oder die Darstellung von Autor, Rezipienten und Auftraggeber als Teile einer geistlichen Solidargemeinschaft.

Bei den vier germanistischen Fallstudien untersucht Maximilian Benz (S. 105-124), was Rudolf von Ems über Gönner und Schreibgründe sagt. Benz sieht hier eine Spannbreite zwischen Heteronomie, also Fremdbestimmtheit, und »kontrolliertem Eigensinn« (S. 108), also der selbstbewussten Vertretung litera- 
rischer Autonomie in den Grenzen des innerhalb einer Textsorte Möglichen. So könnte die inszenierte Klage über die Rezeptionssituation so verstanden werden, dass auf ideale Rezipienten gehofft werde, worunter in diesem Fall Konrad von Winterstetten und sein Kreis zu verstehen wären. Benz plädiert im Grundsätzlichen dafür, soziohistorische Bedingungen nicht im Rahmen eines neuen »Textimmanentismus« (S. 112) zu vernachlässigen. Die politische Funktion der Texte, die er wichtig nimmt, ist dann aber in den religiösen Texten nicht zu finden; diese werden hinsichtlich der Interpretation der Gönnerverhältnisse als weniger ergiebig eingestuft. Für die möglichen Bezüge zu den Kreisen des Bodenseeraums und zu den Staufern weist Benz zahlreiche Optionen auf, deren spekulative Anteile jeweils markiert bleiben.

Seraina Plotke (S. 125-147) behandelt Konrad von Würzburg und den Basler Kreis, bei denen einmal kein Mangel an textexternen Hinweisen herrscht. Urkundliche Überlieferung erlaubt es, die genannten Gönner und Bezugspersonen in Gruppen zu scheiden (Domkapitel, Stadtrat, Adlige, Bürger). In ausführlicher Analyse werden in den Pro- und Epilogen die Angaben zum Auftrag, zu Gönnern und zu deren Beziehungen zum Verfasser sowie die Authentifizierungsstrategien analysiert. Konrad betont sowohl die materielle Unterstützung als auch den literarischen Geschmack des Förderkreises. Er macht deutlich, dass die verschiedenen Instanzen, die zum Entstehen von Großepik gehören, sämtlich von Kennerschaft und Liebe zur Literatur geprägt sind: »Mäzenatentum umschließt Aspekte diviner Intelligibilität genauso wie profaner Materialität« (S. 146). Geistliche wie weltliche Gönner fördern beide Arten von Literatur; die Konzeptualisierung und Darstellung der Beziehungen zwischen Verfasser und Gönnern sind aber textsortenabhängig.

Alan V. Murray (S. 149-166) untersucht Sangsprüche über dänische Herrscher vor dem Hintergrund der Frage, ob diese an ein dänisches Publikum gerichtet gewesen sein konnten. Ausgehend davon, dass Nennungen nicht sofort Gönnerverhältnisse implizieren und dass unbekannt ist, inwieweit Mittelhochdeutsch am dänischen Hof verstanden wurde, favorisiert er andere Auftraggeber sowie Aufführungen im Reich (Reinmar von Zweter) oder vor deutschsprachigem Publikum im dänischen Herrschaftsbereich (Rumelant, Frauenlob).

Christoph Fas benders (S. 167-182) Beitrag zum Deutschen Orden richtet sich auf die Auftragswerke aus der Zeit der Hochmeister Luder von Braunschweig und Dietrich von Altenburg (1331-1341). Fasbender konzentriert sich auf Aspekte, die er als »auftragsrechtlich[ ]« (S. 181) zusammenfasst: Erwägungen über die materiale Gestalt des Textes (wie über die vier zerstörten Quinternionen bei Nikolaus von Jeroschin, der mit ihrer Nennung den Gönnerwechsel gegen Kritik von beiden Seiten abfedert), den Begriff der `Schenkung، bei Tilo von Kulm (die als ausgehandeltes Verhältnis angesehen wird) und Übereinkünfte zum Memorialge- 
denken. Insgesamt sind die Angaben der Texte vage, sie erfordern einen hohen Deutungsaufwand und eine Reihe Mutmaßungen.

Drei Beiträge stammen aus Nachbarphilologien; zwei davon sind ebenfalls Fallstudien. Der anglistische Beitrag von Thomas Haye (S. 185-200) zu Walter von Peterborough zeichnet präzis das historische Feld nach, in das sich Walters Gedicht über die Schlacht von Nájera (1367) einfügt: Konvent, Abt, König, Prinz, Herzog und dessen Kaplan bilden das Beziehungsgefüge, an das sich der Autor richtet. Seine Darstellung wird auf der Grundlage der historischen Rahmenbedingungen, der angenommenen Repräsentationsbedürfnisse und der Erwartungen des Publikums gedeutet.

Der Beitrag zu Mäzenen in der mittelniederländischen Literatur von Remco Sle i d e rin k (S. 201-221) erarbeitet eine breite Forschungsgrundlage. Er rekapituliert zunächst die Forschungsgeschichte und erstellt dann Listen aller mittelniederländischen Texte vom 12. bis späten 15. Jahrhundert, die auf Ersuchen einer benannten Person geschrieben wurden, die namentliche Widmungen tragen oder die auf Ersuchen einer ungenannten Person geschrieben sind. Die erste und die dritte Liste enthalten etwa gleichviel Frauen und Männer; die Widmungen der zweiten Liste beziehen sich nur auf Hochadlige aus Holland und Brabant. Auf der Liste der ungenannten Personen überwiegen fiktionale Texte. Offenbar gab es Mäzenatentum im gesamten Zeitraum; es wird aber auch im niederländischen Bereich nur in einem Bruchteil der Werke, und dann oft nur undeutlich, thematisiert.

In Ariosts ১Orlando Furioso<, so Thomas Kl in kert (S. 223-235), ist der Widmungsempfänger Ippolito d'Este schon im Titel des Erstdrucks klar benannt und wird in die Erzählung, die ihm von seiner Genealogie berichten soll, einbezogen. Das Herrscherlob im Text wird als Teil einer Strategie des Autors eingestuft, die Reputation und Nachruhm begründet. Diffizil wird die Analyse aufgrund der ironischen Sprechhaltung, die wunderbare Aporien liefert, wenn beispielsweise die Kritik an unzuverlässigen Autoren im Text ausgerechnet vom Evangelisten Johannes vorgetragen wird.

Zwei Beiträge folgen schließlich zu anderen Fächern: Die Kunstgeschichte repräsentiert Klaus Gereon Beuckers (S. 239-266) Artikel zu ottonischer und frühsalischer Buchmalerei. Die umfassende, sehr plastische Analyse von Herrscherbildern des 10. und 11. Jahrhunderts (aus Echternach, der Reichenau und Köln) macht wahrscheinlich, dass diese nicht im Auftrag der Abgebildeten entstanden, sondern von geistlichen Skriptorien erstellt wurden, um auf diese Herrscher einzuwirken. In Konsequenz werden damit gerade die abgebildeten Personen aus dem Thema >Mäzenatentum ‘ herausgedrängt; an ihre Stelle treten meist anonyme Kleriker, von denen wir kaum etwas wissen - außer ihren verbildlichten Konzeptionen von Herrschaft. 
Während Andreas Bihrers Beitrag aus geschichtswissenschaftlicher Perspektive den Band bei den Grundlagen eröffnen durfte, findet sich Klaus O s c h e m a s (S. 267-291) Fallstudie zu spätmittelalterlichen Prognostiken unter den disziplinären Erweiterungen am Schluss. Diese von Astrologen erstellten Vorschauen auf das kommende Jahr werden oft von Widmungen begleitet; sie erfüllen auch politische und verbreitungsstrategische Zwecke. Oschema zeichnet nach, wie sich die Autoren über teils persönliche Kenntnisse den Widmungsträgern annähern; es wird aber auch festgestellt, dass der Handel, der mit der Titelzeile >Zukunft gegen Patronage?^ nahegelegt wird, aus den Texten nicht wirklich zu belegen ist.

Betrachtet man den gesamten Band, dann erklingt ein starker Grundbass, der zum einen die schmale Quellenlage und zum anderen ihre begrenzte Aussagekraft oder Aussagebereitschaft beklagt. Wie dünn die Faktenlage ist, zeigt sich etwa im Abgleich mit den wesentlich häufigeren Autornennungen (bei Kipf), aber auch in der Bereitschaft der Beiträger, sämtlichen im Text genannten Personen nachzuforschen. Ein weiteres Indiz ist die explizite Unsicherheitserklärung: sechs ungelöste Fragen gibt Benz an (vgl. S. 123 f.); Bihrer hat die Ungelöstheit gar zum Strukturprinzip gemacht; in seinem Beitrag zähle ich fast 60 Fragezeichen. Weitere Anregungen für künftiges Forschen benennt die ১Einleitung` (vgl. S. 21).

Der Band spornt also einerseits an, andererseits zeigt er in beeindruckender Weise, dass genaues Hinschauen unter modifizierten Aspekten allemal lohnt. Dabei ist festzustellen, dass alle konkreten Beispiele immer auch einen guten Teil ihrer Bemühung auf die historische Situierung legen und legen müssen. Das bringt viele hochinteressante, auch für die Geschichtswissenschaft auswertbare Details ans Tageslicht, die hier notwendigerweise zurückstehen mussten, welche die Nutzer aber über die Register (S. 293-302) auffinden mögen. Dort, wo die Fragen der Literarisierungsstrategien in den Vordergrund treten, muss häufiger zu Mutmaßungen gegriffen werden.

Es folgen noch drei Bemerkungen zum Titel. Die Zuordnung zu der mit >Mittelalter benannten Kernzeit ist beim Beitrag zum 〉Orlando furioso (1516-1532) nicht zwingend. Die offene Frage, wie Paratexte, Druckauflagen oder durchgehende selbstreferentielle Ironisierung zu den im übrigen Band beschriebenen Verhältnissen des Hoch- und Spätmittelalters stehen, wird allerdings nirgends gestellt. Die seuropäische Perspektive « kann in künftiger Forschung noch intensiviert werden. Dass Bumkes Alliteration >Mäzene im Mittelalter` dazu geführt hat, für mittelalterliche Gönner einen Begriff $\mathrm{zu}$ etablieren, der eigentlich eine vom Einzelwerk unabhängige Künstlerförderung bezeichnet und sich erst seit dem 19. Jahrhundert durchsetzt, wird in der Einleitung angesprochen: >Mäzen ‘ soll im Band wie bei Bumke die gesamte Bandbreite von Auftraggeberschaft bis Kulturförderung umfassen (vgl. S. 13). Wieso dann im Titel versucht wird, mit den `Mäzenaten` (swm.[?]) einen anderen, verwandten Begriff zu etablieren, ist nicht 
erklärt. >Mäzenat` (stn.) wäre eigentlich das Verhältnis zwischen Mäzen und Künstler; von den Beiträgern wird der Begriff dann auch nicht übernommen.

Unter einer möglichst breiten Vorstellung von `Mäzen` werden im Band alle denkbaren Bezüge entfaltet; das kann vom theologischen Berater und wohlwollenden Freund bis zum Übersetzer reichen. Insgesamt deutet der Band geradezu auf eine Armada von Leuten, die zur Textgenese gehören und weder Autor noch Publikum sind. Gemeinsam ist ihnen, dass sie allesamt schwer zu fassen sind. Da wir davon ausgehen dürfen, dass Mäzene im Mittelalter von grundlegender Wichtigkeit sind, ist es mehr als lästig, dass viele Texte gänzlich versäumen, Hinweise auf sie zu geben.

Andreas Kurzmann: Die deutschen geistlichen Dichtungen. Speculum humanae salvationis - Soliloquium Mariae cum Jesu - De quodam moriente, hg. v. Peter Wiesinger u. Edeltraud Weißenböck-Paflik, Wien: Praesens 2017, 453 S.

Besprochen von PD Dr. Wolfgang Beck: Friedrich-Schiller-Universität Jena, Institut für Germanistische Literaturwissenschaft, Fürstengraben 18, D-07743 Jena, E-Mail: wolfgang. beck@uni-jena.de

https://doi.org/10.1515/bgsl-2018-0047

Andreas Kurzmann († vor 1431), Mönch im Zisterzienserkloster Neuberg an der Mürz (Steiermark), ist Verfasser von fünf volkssprachigen religiösen Dichtungen. Bereits ediert sind die Inzestlegende `St. Alban ${ }^{1}$ (`SA`, 923 Verse) und die Freundschaftslegende >Amicus und Amelius ${ }^{2}$ (`AA`, 1165 Verse), die in dieser Ausgabe nicht berücksichtigt wurden. Alte Ausgaben existieren von der Erlösungsdichtung >Soliloquium Mariae cum Jesu ${ }^{3}$ (`SO২, 427 Verse) und dem Streitgespräch $>$ De quodam moriente $\iota^{4}$ ( $>\mathrm{MO}<, 91$ Verse), die durch die hier vorliegende ersetzt werden. Das dem Umfang nach größte Werk Kurzmanns, eine deutsche

1 Karin Morvay: Die Albanuslegende. Deutsche Fassungen und ihre Beziehungen zur lateinischen Überlieferung, München 1977 (Medium Aevum 32), S. 92-116.

2 Christine Manuela Rainer: Die Legende >Amicus und Amelius` des Andreas Kurzmann (Salzburg, UB, Cod. M I 138). Mehrschichtige Edition, stoffgeschichtliche Einordnung und Glossar, Masterarbeit, Graz 2012 (URL: http://unipub.uni-graz.at/download/pdf/216979 [Aufrufdatum: 10.10.2018]).

3 Anton Schönbach: Über die Marienklagen. Ein Beitrag zur Geschichte der geistlichen Dichtung in Deutschland, Festschrift der k. k. Universität in Graz zur Jahresfeier am 15. November 1874, Graz 1874, S. 73-83. Wieder abgedruckt bei Hedwig Heger (Hg.): Spätmittelalter - Humanismus - Reformation. Texte und Zeugnisse. Bd. 1, München 1975, S. 3-9.

4 Josef Ampferer: Der Mönch von Salzburg. In: Vierzehntes Programm des kaiserlich königlichen Staats-Gymnasiums in Salzburg, Salzburg 1864, S. $31 \mathrm{f}$. 


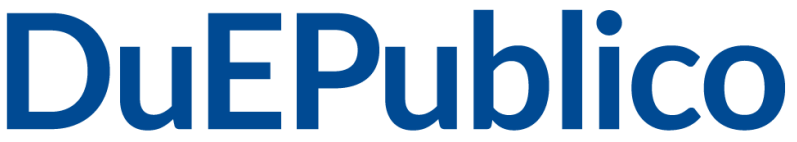

Duisburg-Essen Publications online
UNIVERSITÄT

DE $\mathrm{U}_{S}$ IS $_{\mathrm{E}} \mathrm{B}_{\mathrm{N}} \mathrm{U} R \mathrm{R}$

Offen im Denken

Ub universitäts

Dieser Text wird über DuEPublico, dem Dokumenten- und Publikationsserver der Universität Duisburg-Essen, zur Verfügung gestellt. Die hier veröffentlichte Version der EPublikation kann von einer eventuell ebenfalls veröffentlichten Verlagsversion abweichen.

DOI: $\quad 10.1515 /$ bgsl-2018-0046

URN: urn:nbn:de:hbz:464-20200529-110219-4

Alle Rechte vorbehalten.

Dieser Beitrag ist mit Zustimmung des Rechteinhabers aufgrund einer (DFG-geförderten) Allianz- bzw. Nationallizenz frei zugänglich. 